\title{
Replication Stress and Telomere Dysfunction Are Present in Cultured Human Embryonic Stem Cells
}

\author{
Christine Janson Kristine Nyhan John P. Murnane \\ Department of Radiation Oncology, University of California San Francisco, San Francisco, Calif., USA
}

\section{Key Words}

Activin A · Human embryonic stem cells · Replication stress . Telomere

\begin{abstract}
Replication stress causes DNA damage at fragile sites in the genome. DNA damage at telomeres can initiate breakagefusion-bridge cycles and chromosome instability, which can result in replicative senescence or tumor formation. Little is known about the extent of replication stress or telomere dysfunction in human embryonic stem cells (hESCs). hESCs are grown in culture with the expectation of being used therapeutically in humans, making it important to minimize the levels of replication stress and telomere dysfunction. Here, the hESC line UCSF4 was cultured in a defined medium with growth factor Activin A, exogenous nucleosides, or DNA polymerase inhibitor aphidicolin. We used quantitative fluorescence in situ hybridization to analyze individual telomeres for dysfunction and observed that it can be increased by aphidicolin or Activin A. In contrast, adding exogenous nucleosides relieved dysfunction, suggesting that telomere dysfunction results from replication stress. Whether these findings can be applied to other hESC lines remains to be determined. However, because the loss of telomeres can
\end{abstract}

lead to chromosome instability and cancer, we conclude that hESCs grown in culture for future therapeutic purposes should be routinely checked for replication stress and telomere dysfunction.

(c) 2015 S. Karger AG, Basel

Telomeres consist of a TTAGGG repeat sequence found at the ends of chromosomes. The shelterin complex of proteins binds to this repeat sequence, providing a protective cap that prevents chromosome ends from being recognized as DNA double strand breaks (DSBs) [Nandakumar and Cech, 2013]. DSBs near telomeres are particularly important because when a telomere is lost or damaged, the cell can undergo chromosome fusions, resulting in breakage-fusion-bridge cycles leading to malignant transformation [Lo et al., 2002]. Fumagalli et al. [2012] showed that following treatment of human primary fibroblasts with ionizing radiation, DSBs near telomeres persist long after DSBs at interstitial sites have been repaired. This study also found that these persistent telomeric DSBs are associated with ionizing radiation-induced replicative senescence. Our laboratory has also demonstrated that telomeric regions are highly sensitive to DSBs, and that this sensitivity to DSBs can result in

\section{KARGER 125}

(c) 2015 S. Karger AG, Basel

1424-8581/15/1464-0251\$39.50/0

E-Mail karger@karger.com

www.karger.com/cgr 
chromosome instability in cancer cells [Sabatier et al., 2005; Muraki et al., 2012].

Telomere dysfunction can occur due to gradual shortening of telomeres in cells lacking telomerase [d'Adda di Fagagna et al., 2003; Capper et al., 2007]. However, telomere dysfunction can also occur in telomerase-positive cells due to stochastic events resulting from replication stress that can occur following the disruption or partial inhibition of DNA synthesis [Sfeir et al., 2009; Suram et al., 2012]. Replication stress causes DSBs at fragile sites [Zeman and Cimprich, 2014], which are DNA sequences in the genome that have slower rates of replication. Due to oncogene-induced replication stress, fragile sites, like telomeres, are hotspots for chromosome rearrangements in cancer cells [Bignell et al., 2010]. In view of the fact that DSBs near telomeres are poorly repaired, we have previously proposed that those resulting from replication stress are an important mechanism for genomic instability in cancer [Murnane, 2010]. Consistent with this proposal, reducing replication stress has been shown to decrease the tumorigenicity of cancer cells [Bester et al., 2011]. Briefly, replication stress induces telomere dysfunction, which can lead to either replicative senescence or tumorigenesis depending on the cell type involved.

In the current study we investigated telomere dysfunction in a human embryonic stem cell (hESC) line, UCSF4, using quantitative fluorescence in situ hybridization (QFISH) to determine how the growth in culture affects replication stress. In view of the consequences of telomere dysfunction for chromosome instability and the fact that cells derived from hESCs are intended for use in humans, understanding how culture conditions influence replication stress and telomere dysfunction in hESCs is of high importance. Our results show that culturing conditions can influence telomere dysfunction in the hESC line UCSF4, and that monitoring telomere dysfunction by QFISH is an effective way of optimizing culture conditions for hESCs.

\section{Materials and Methods}

\section{Cell Culture and Reagents}

The hESC line UCSF4 was derived by Dr. Susan Fisher (UCSF), NIH registry number 0044 [Krtolica et al., 2011; Felfly and Klein, 2013]. UCSF4 was grown in feeder-free mTeSR media (STEMCELL Technologies) with either Matrigel (Corning) or Geltrex basement membrane (Life Technologies). ROCK inhibitor (10 $\mu \mathrm{M}$, Selleckchem) was added to the cultures during passaging and was removed after $24 \mathrm{~h}$. Activin A (10 ng/ml, R\&D Systems), IGF (200 ng/ml, LONG R3 IGF-1, Sigma), and nucleosides (EmbroMax Nu- cleosides, Millipore) were added to cell cultures for 5 days before analysis. Aphidicolin (Fisher Scientific) treatment was performed by allowing the cells to attach and grow for $24 \mathrm{~h}$, after which 0.1 or $0.2 \mu \mathrm{M}$ aphidicolin was added for $16 \mathrm{~h}$, followed by a 2 -hour release from the drug.

\section{Q-FISH}

Q-FISH for telomere analysis was performed as previously described [Poon and Lansdorp, 2001]. Briefly, cells were arrested in metaphase by incubation with colcemid (Invitrogen) for $\sim 3 \mathrm{~h}$. Cells were pelleted, washed once in PBS, and resuspended in hypotonic buffer (Genial Genetics). After $20 \mathrm{~min}$ incubation at $37^{\circ} \mathrm{C}$, cells were fixed in 3:1 methanol:acetic acid and dropped onto slides in a humid chamber. After drying overnight, slides were fixed in $4 \%$ formaldehyde, treated with $1 \mu \mathrm{g} / \mathrm{ml}$ pepsin (Sigma), fixed again in $4 \%$ formaldehyde, and dehydrated in decreasing amounts of ethanol. A PNA probe specific for telomere repeat sequences (TelC-Cy3 from PNA Bio) was hybridized to the samples by heating slides to $80^{\circ} \mathrm{C}$ for $3 \mathrm{~min}$ followed by a 2-hour incubation in a humid chamber at room temperature. Slides were then extensively washed and dehydrated in ethanol, and after drying overnight, coverslips were applied to the slide with Prolong Gold anti-fade reagent with DAPI (Invitrogen).

\section{Immunofluorescent Staining}

For immunofluorescent staining, cells were grown on coverslips, fixed in $2 \%$ paraformaldehyde in interphase, washed in PBS, and permeabilized in $80 \%$ methanol. Samples were blocked with $0.1 \%$ BSA and incubated with primary antibody to 53BP1 (1:200, Bethyl Laboratories), followed by goat anti-rabbit AlexaFluor 488. Coverslips were briefly fixed again and blocked with $1 \%$ BSA. Cells were stained with OCT4 primary antibody (1:400, sc-5279, Santa Cruz) and goat anti-mouse AlexaFluor 647. Immunofluorescent staining was followed by PNA staining for telomere repeats. Coverslips were applied to the slides with Prolong Gold anti-fade reagent with DAPI (Invitrogen).

\section{Microscopy}

Metaphase spreads were imaged on a Zeiss Axio Imager Z2 upright microscope using a Metasystems Metafer slide-scanning platform. Images were taken at $\times 63$ using Metafer software. Telomere dysfunction-induced foci (TIF) images were obtained on a Zeiss AxioImager 2 multi-channel, upright microscope at $\times 63$ magnification using Axiovision software.

\section{Growth Curves}

Cells were plated at $2 \times 10^{5}$ cells/well in 6 well plates. At the days indicated, the cells were trypsinized and resuspended with $0.04 \%$ trypan blue to detect and exclude dead cells. Each well was counted in duplicate on a Nexcelom Cellometer.

\section{Cell Cycle Analysis}

Cells were trypsinized, washed twice in PBS containing $0.1 \%$ $\mathrm{BSA}$, and resuspended at $1 \times 10^{6}$ cells $/ \mathrm{ml}$. While gently vortexing, $3 \mathrm{ml}$ of cold $100 \%$ ethanol was added drop wise to $1 \mathrm{ml}$ of cells. After overnight incubation at $4^{\circ} \mathrm{C}$, the cells were washed and resuspended in $1 \mathrm{ml}$ of PBS containing $0.1 \% \mathrm{BSA}, 40 \mu \mathrm{g} / \mathrm{ml}$ propidium iodide, and $500 \mu \mathrm{g} / \mathrm{ml}$ RNAse A. After a 3-hour incubation period at $4^{\circ} \mathrm{C}$, the cells were analyzed using an Accuri C6 flow cytometer and C-Flow software. 
Telomerase Assay

Telomerase activity was determined with the TRAPeze XL Telomerase Detection Kit (Millipore) using the manufacturer's instructions. The TRAPeze XL Kit detects telomerase activity in cell extracts by first performing telomere extension on a template, which is then amplified by PCR. The extent of amplification is detected by fluorometric analysis using Amplifluor primers. Telomerase activity is then determined by the ratio of fluorescein to sulforhodamine emission based on amplification of the internal control template TSK2. Negative controls consist of the same extracts heated at $85^{\circ} \mathrm{C}$ for $10 \mathrm{~min}$ to inactivate telomerase activity. The positive control consists of an extract made from $10^{6}$ telomerase-positive cells supplied with the kit.

\section{Results}

\section{Measuring Telomere Dysfunction in UCSF4 hESCs}

The hESC line UCSF4 was analyzed for telomere dysfunction; all experiments were performed with cells between passage number 20 and 40 . The level of telomerase activity was high and remained stable in UCSF4 cells during this time in culture (fig. 1). Dysfunctional telomeres were identified using Q-FISH, which utilizes a PNA probe specific for telomere repeat sequences. Healthy telomeres appear as round, punctate signals at the ends of the chromatids in mitosis. Dysfunctional telomeres were placed in 4 categories, examples of which are shown in figure 2 . These categories include telomere signals that are smeared, telomeres that appear as doublets, undetectable telomeres, and short telomeres on both sister chromatids. Short telomeres were identified as those where staining was detectable but significantly dimmer than the average for the cell. Other research groups have shown that telomere dysfunction detected by Q-FISH is associated with DNA damage, as identified by TIFs [Takai et al., 2003]. TIFs are identified by co-localization of a telomere with a DSB-associated protein [Takai et al., 2003; Sfeir et al., 2009] such as 53BP1, demonstrating that telomere dysfunction is related to DSBs. We observed that the UCSF4 cell line grown in defined mTESR1 medium has an average of 7 dysfunctional telomeres per cell (fig. 3a).

\section{Aphidicolin-Induced Replication Stress Increases}

Telomere Dysfunction in UCSF4 hESCs

Aphidicolin is an inhibitor of DNA polymerase. Although high levels of aphidicolin cause cell death, lower levels cause replication stress, leading to stalled replication forks at fragile sites [Arlt et al., 2012]. Because telomeres are fragile sites, aphidicolin-induced replication stress results in telomere dysfunction [Martínez et al.,

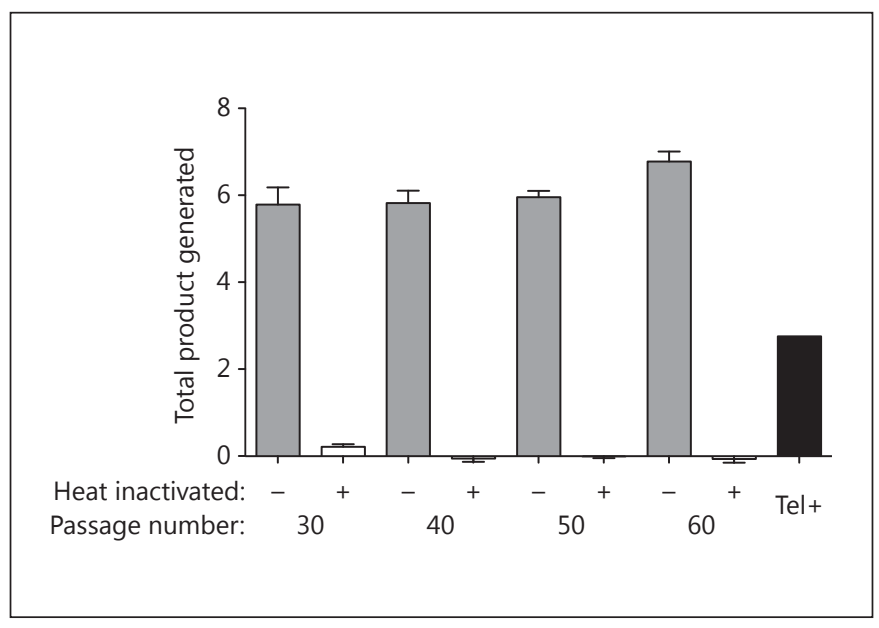

Fig. 1. Telomerase activity in UCSF4 hESCs at various passage numbers. Extracts from UCSF4 cells were assayed for telomerase activity using the TRAPeze XL Telomerase Detection Kit at passage 30, 40, 50 and 60 . Negative controls consist of heat-treated extracts. A positive control consists of extract made from $10^{6}$ telomerase-positive cells included in the kit (Tel+). The level of telomerase activity is given as total product generated, which is calculated by determining the ratio of amplification of the newly added telomeric repeat sequences (fluorescein) to the amplification of the internal TSK2 control template (sulforhodamine).

2009; Sfeir et al., 2009]. UCSF4 cells treated overnight with low levels of aphidicolin showed increased dose-dependent telomere dysfunction compared to DMSO controls (fig. 3a). Aphidicolin treatment also increased the percent of cells with $53 \mathrm{BP} 1$ foci (fig. $3 \mathrm{~b}$, e) as well as the percent of cells with more than one 53BP1 foci (fig. 3c). With DMSO-treated control populations, $15.4 \%( \pm 3.3 \%$ SEM) of the cells had 53BP1 foci, while 0.1 and $0.2 \mu \mathrm{M}$ aphidicolin increased the percent of cells with 53BP1 foci to $38.7 \%( \pm 5.7 \%$ SEM $)$ and $64.1 \%( \pm 11.9 \%$ SEM $)$, respectively ( $\mathrm{p}<0.05)$. In addition, the percent of 53BP1-positive cells with 4 or more foci increased from $7.2 \%( \pm 0.2 \%$ SEM) in DMSO control populations to $18.6 \%( \pm 3.1 \%$ SEM) and $22.5 \%( \pm 4.5 \%$ SEM $)$ in 0.1 and $0.2 \mu \mathrm{M}$ aphidicolin-treated populations, respectively $(\mathrm{p}<0.05$ for both concentrations). TIF formation also increased with aphidicolin treatment. However, while the percent of cells with TIFs increased (fig. 3d), the number of TIFs per cell did not significantly change (data not shown). This is primarily due to the fact that TIFs were usually present only at 1 TIF per cell (fig. 3e). Therefore, similar to other cultured cells, aphidicolin causes telomere dysfunction in UCSF4 cells. 


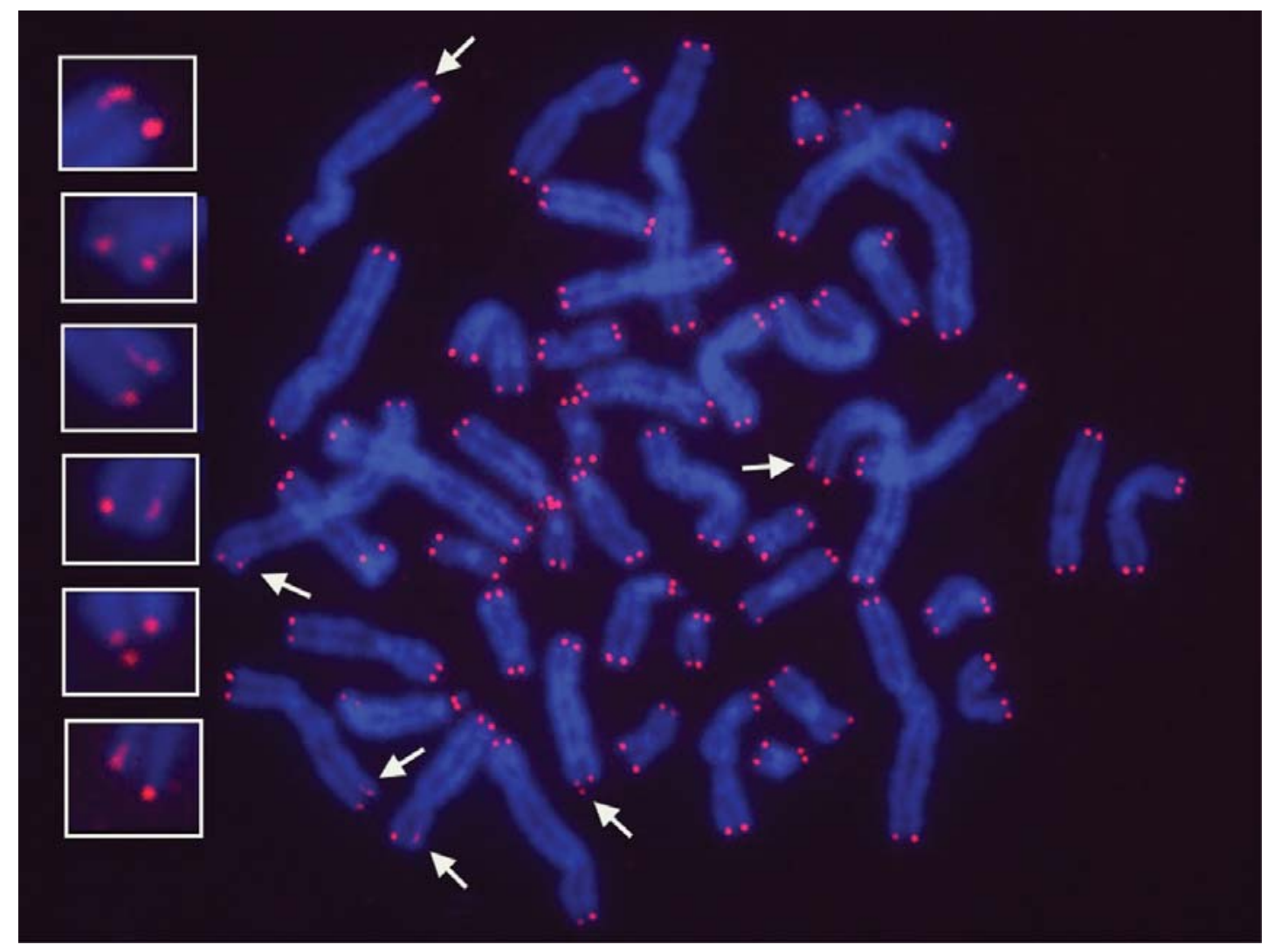

Fig. 2. Telomere dysfunction in the UCSF4 hESC line. Examples of dysfunctional telomeres are shown in the insets. Telomeric repeat sequences were stained with a Cy3-labeled PNA probe (red), and total DNA was stained with DAPI (blue). Magnification: $\times 100$.

\section{Addition of Exogenous Nucleosides Decreases}

\section{Telomere Dysfunction in UCSF4 hESCs}

One well-documented cause of replication stress is the depletion of nucleotide pools, as shown by the extensive replication stress caused by hydroxyurea, which reduces nucleotide synthesis by inhibiting the enzyme ribonucleotide reductase [Arlt et al., 2012; Zeman and Cimprich, 2014]. Nucleosides are readily taken up by cells in culture and are precursors of deoxynucleotide triphosphates that are incorporated into the new strand during DNA synthesis. If DNA polymerase does not have access to sufficient deoxynucleotide triphosphates, it will stall, which can result in a DSB [Zeman and Cimprich, 2014]. Addition of exogenous nucleosides can relieve DNA damage due to replication stress in cancer cells, showing that replication stress associated with cancer is at least partially due to insufficient nucleotide pools [Burrell et al., 2013; Bester et al., 2011]. To determine whether replication stress and telomere dysfunction in UCSF4 cells are also associated with insufficient nucleotide pools, we tested whether the addition of nucleosides to the growth medium can reduce replication stress. The addition of 15 and $30 \mu \mathrm{M}$ nucleosides to the growth medium reduced dysfunctional telomeres from an average of $7.8 \pm 0.7$ per cell to an average of $5.7 \pm$ 0.3 and $5.9 \pm 0.3$ per cell, respectively $(\mathrm{p}<0.05)$ (fig. $4 \mathrm{a})$. The addition of nucleosides caused no significant change in the percent of cells with 53BP1 foci (fig. 4b). However, the addition of nucleosides did reduce the number of 53BP1 foci per cell (fig. $4 \mathrm{c})$. A total of $7.1 \%( \pm 0.24 \%$ SEM) of the untreated control cells with 53BP1 foci had 4 or more foci, while only $2.8 \%( \pm 0.04 \%$ SEM $)$ of the 53BP1-positive nucleoside-treated cells had 4 or more foci $(\mathrm{p}<0.001)$. Additionally, nucleosides decreased TIF formation (fig. 4d). Untreated control cells showed 3.9\% $( \pm 0.2 \%$ SEM) TIFpositive cells, while the addition of nucleosides reduced TIF-positive cells to $2.8 \%( \pm 0.2 \% \mathrm{SEM})(\mathrm{p}<0.05)$. Increasing nucleotide pools therefore decreases dysfunctional telomeres associated with DNA damage in UCSF4 cells. 
Fig. 3. Aphidicolin induces telomere dysfunction in the UCSF4 hESC line. a Quantification of dysfunctional telomeres in cells treated with DMSO, or 0.1 or $0.2 \mu \mathrm{M}$ aphidicolin. $\mathbf{b}$ Percentage of the population with 53BP1 foci in cells treated with DMSO, or 0.1 or $0.2 \mu \mathrm{M}$ aphidicolin. c Stack graph represents the percent of 53BP1-positive cells with $1,2,3$, or $4+$ foci per cell. d Percentage of the population with TIF foci. Bars in a-d represent the mean \pm SEM of 3 independent experiments. Student's 2 -tailed t test was used to determine significance: ${ }^{*} \mathrm{p}<0.05,{ }^{* *} \mathrm{p}<0.01$. e Fluorescent imaging of cells treated with DMSO or $0.2 \mu \mathrm{M}$ aphidicolin. Cells were stained for 53BP1 (green), telomere repeat sequences (red), and total DNA (blue). Arrowheads indicate the location of DSBs. Magnification: $\times 60$.

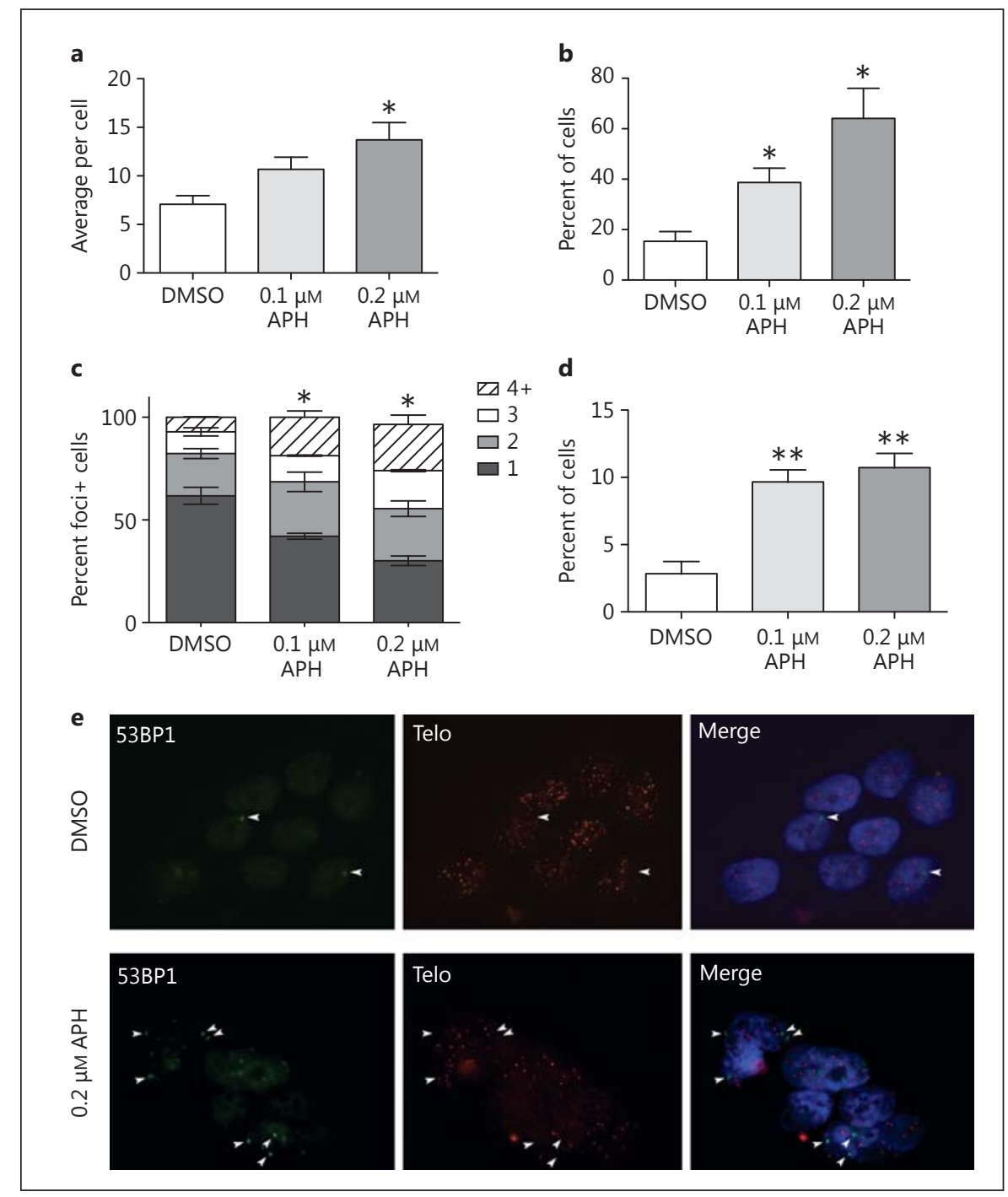

\section{Addition of Growth Factor Activin A Induces}

Telomere Dysfunction in UCSF4 hESCs

hESCs grown in feeder-free, unconditioned defined medium require fibroblast growth factor to maintain pluripotentency and remain actively dividing [Xu et al., 2005]. Additional growth factors are also commonly added to hESC growth media to enhance proliferation and maintain pluripotency. One such growth factor is Activin A, a member of the tumor growth factor beta (TGF- $\beta$ ) family. Other laboratories have previously reported that when added at low levels $(10 \mathrm{ng} / \mathrm{ml})$, Activin A maintains hESC proliferation and pluripotency by signaling through SMAD proteins [Beattie et al., 2005; Xiao et al., 2006]. To determine their influence on telomere dysfunction, we cultured cells in the presence of Activin

Telomere Dysfuntion in Human

Embryonic Stem Cells
A or insulin-like growth factor-1 (IGF-1) $(200 \mathrm{ng} / \mathrm{ml})$ [Wang et al., 2007]. Addition of Activin A to the growth medium increased the number of dysfunctional telomeres in UCSF4 cultures by $40 \%$ ( $\mathrm{p}<0.05$ ), while the addition of IGF-1 had no effect (fig. 5a). 53BP1 foci (fig. 5b) more than doubled with Activin A treatment, with $53 \mathrm{BP} 1$ foci increasing from $15.4 \%( \pm 3.9 \%$ SEM) to $40.0 \%( \pm 3.6 \%$ SEM $)(p<0.01)$. However, Activin A treatment did not significantly affect the number of 53BP1 foci per cell (fig. 5c). Similar to 53BP1, Activin A treatment also increased TIFs (fig. $5 \mathrm{~d}$ ), which increased from $3.6 \%( \pm 0.2 \%$ SEM $)$ to $8.0 \%( \pm 2.1 \%$ SEM $)(\mathrm{p}<0.05)$. Taken together, these results demonstrate that Activin A increases DNA damage and telomere dysfunction in UCSF 4 cells. 
Fig. 4. Exogenous nucleosides reduce telomere dysfunction in the UCSF4 hESC line. a Quantification of dysfunctional telomeres in UCSF4 cells grown in medium containing exogenous nucleosides. b Quantification of cells with 53BP1 foci with or without exogenous nucleosides. c Stack graph represents the percent of 53BP1-positive cells with $1,2,3$, or $4+$ foci per cell. d Quantification of cell with TIF foci when cultured with or without exogenous nucleosides. Bar graphs represent the mean \pm SEM of at least 3 independent experiments. Student's 2tailed $t$ test was used to determine significance: ${ }^{*} \mathrm{p}<0.05$.

Fig. 5. The growth factor Activin A increases telomere dysfunction in the UCSF4 hESC line. a Quantification of dysfunctional telomeres in UCSF4 hESCs cultured with Activin A or IGF-1, and in control cultures. b Quantification of cells with 53BP1 foci in control and Activin A-treated conditions. c Stack graph represents the percent of 53BP1-positive cells with 1, 2, 3, or 4+ foci per cell. d Quantification of cells with TIFs when cultured with or without Activin A. Bar graphs represent the mean \pm SEM of at least three independent experiments. Student's 2-tailed t test was used to determine significance: ${ }^{*} \mathrm{p}<0.05,{ }^{* *} \mathrm{p}<$ 0.01 .

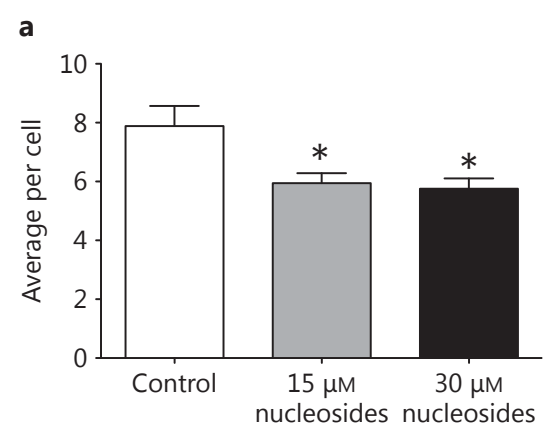

b
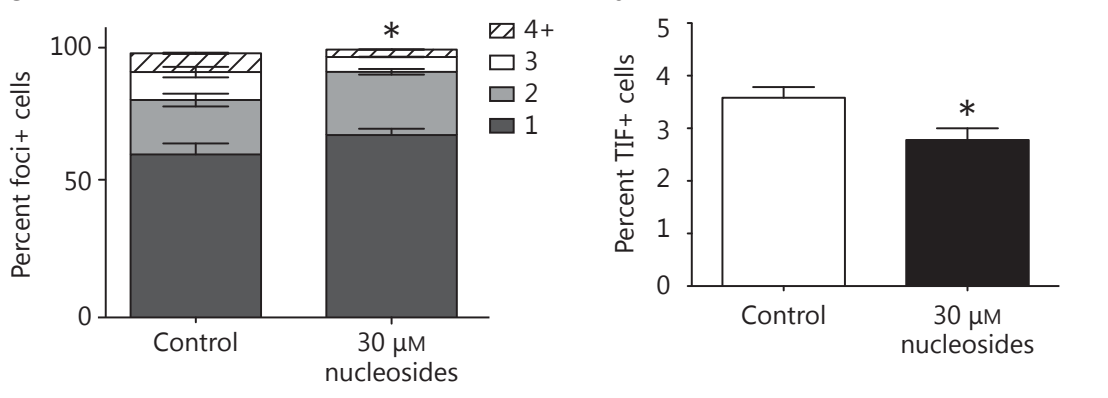

a

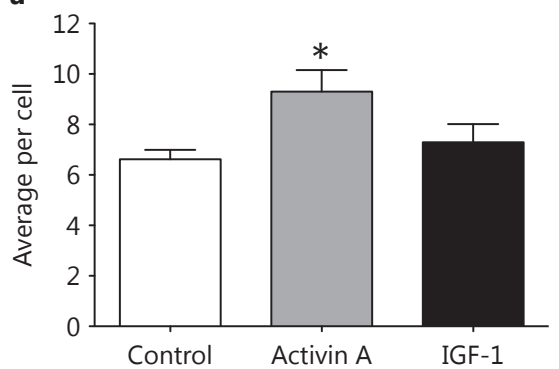

c

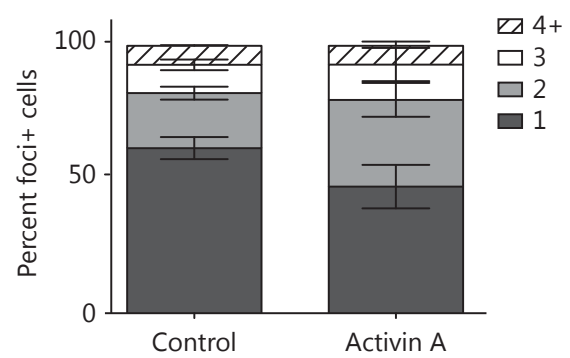

d

b

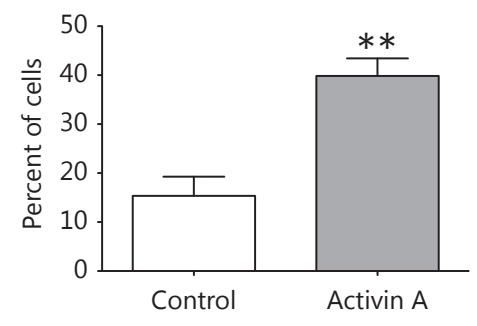

d
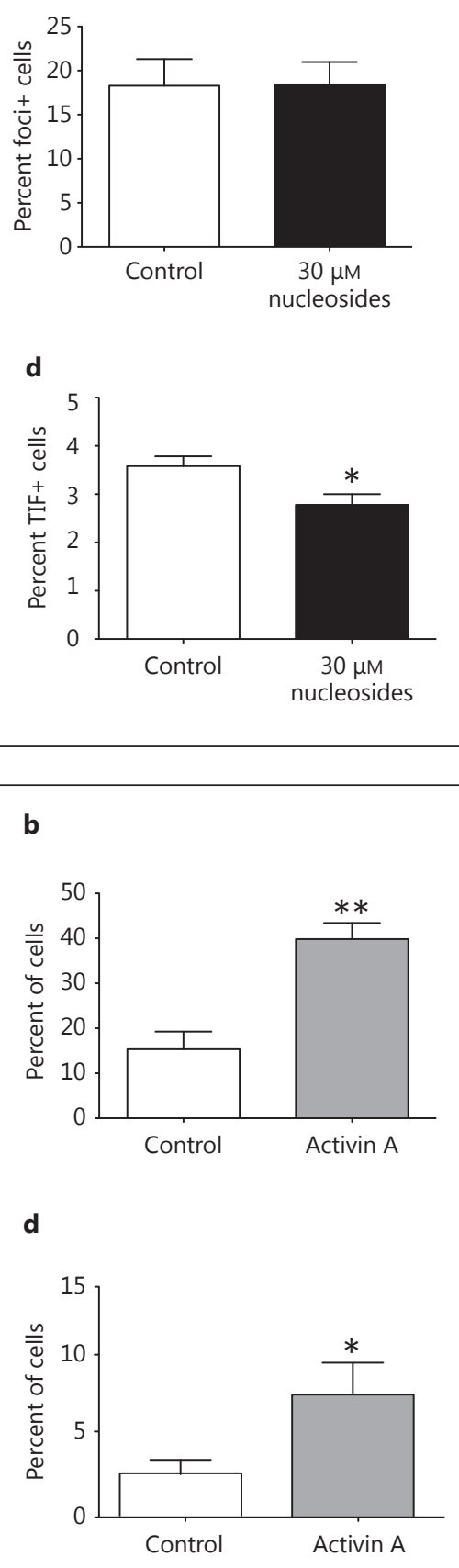

\section{Activin A Causes UCSF4 hESCs to Undergo}

Differentiation and Growth Arrest

Because previous studies found that Activin A helps to maintain proliferation and pluripotency of hESCs [Beattie et al., 2005; Vallier et al., 2005], we investigated the effects of Activin A on growth of UCSF4 cells. Growth curves were performed on UCSF4 cultures grown in regular medium, medium supplemented with Activin A, or medium containing nucleosides (fig. 6a). Addition of nucleosides alone did not significantly change the growth rate of the UCSF4 cultures ( $p>0.09$ for all time points). However, the addition of Activin A severely decreased the 


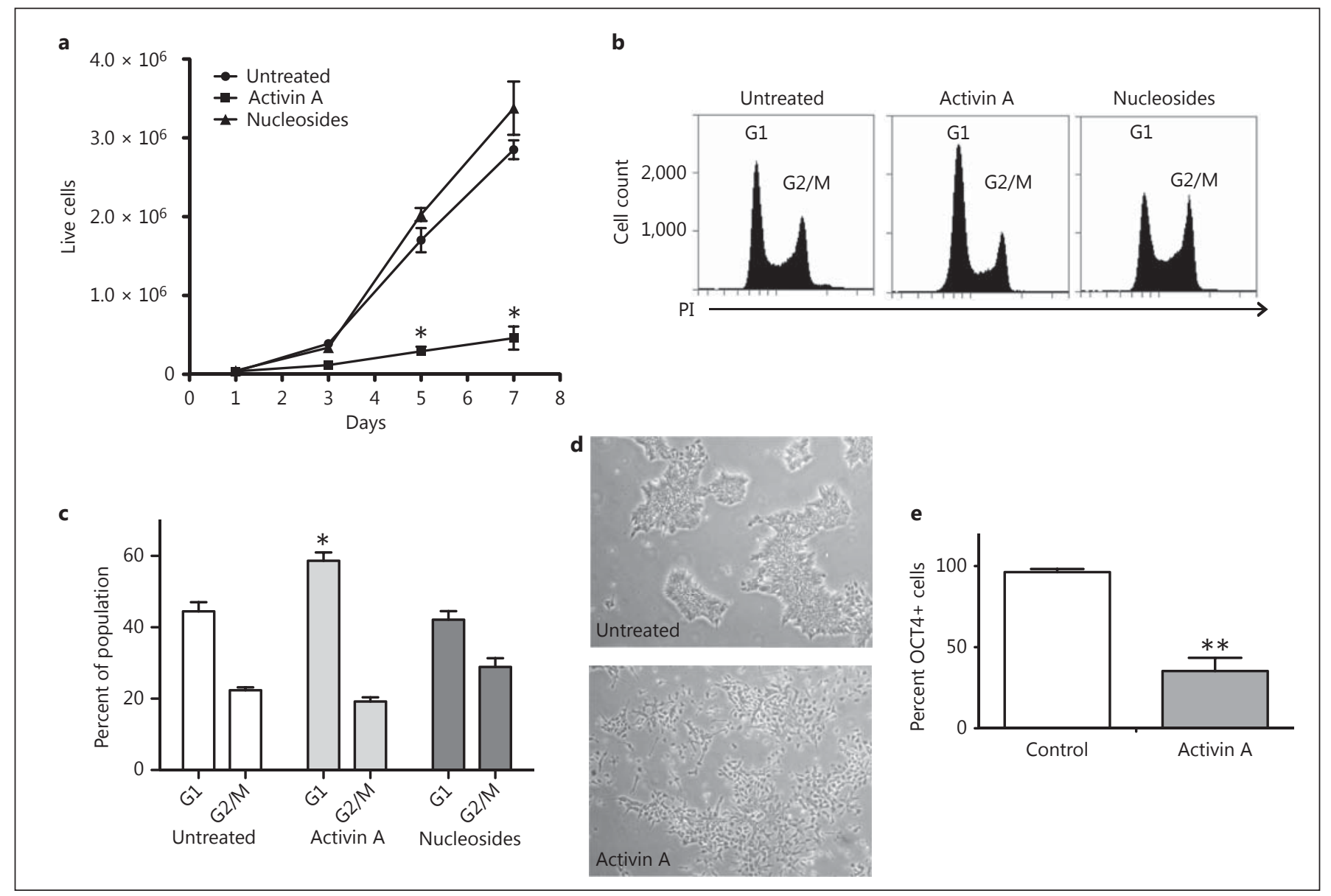

Fig. 6. UCSF4 cells undergo differentiation and growth arrest when cultured with Activin A. a Growth curves for UCSF4 cells grown with Activin A or exogenous nucleosides and in control cultures. b Representative histograms showing cell cycle distribution as measured by propidium iodide staining and flow cytometry for UCSF4 cells grown with Activin A or exogenous nucleosides and in control cultures. c Bar graph of cell cycle distribution for UCSF4 cells grown with Activin A or exogenous nucleosides and in control cultures. $\mathbf{d}$ Cell morphology imaged by brightfield microscopy at $\times 10$ magnification of UCSF 4 cells grown in the presence or absence of Activin A. e Quantification of OCT4 immunofluorescent staining of UCSF4 cells grown in the presence or absence of Activin A. Bar graphs represent mean \pm SEM of 3 independent experiments. Student's 2-tailed t test was used to determine significance: ${ }^{*} \mathrm{p}<0.05,{ }^{* *} \mathrm{p}<0.01$.

compared to controls, the addition of nucleosides to the growth medium resulted in a slight decrease in the percentage of cells in G1 phase $(42.1 \pm 2.4 \%$ SEM $)$ and an increase in the percentage of cells in G2/M (28.9 $\pm 2.5 \%$ SEM).

Next, we explored the reason for the increase in cells in G1 phase caused by Activin A. Undifferentiated hESCs have a weak G1/S checkpoint [Sokolov and Neumann, 2012] and therefore would not be expected to arrest in G1 in response to DNA damage. However, we observed differences in morphology between UCSF4 cultures grown with or without Activin A. The UCSF4 cells in the control cultures grew in typical colonies with rounded morphol- 
Fig. 7. Activin A-treated undifferentiated UCSF4 cells are more likely to have TIFs. a Staining for OCT4, 53BP1, telomere repeat sequences, and total DNA in UCSF4 cells treated with Activin A. Yellow arrowheads indicate OCT4-positive undifferentiated cells, and white arrowheads indicate OCT4-negative differentiated cells. b Quantification of OCT4-positive undifferentiated cells (OCT4+) and OCT4-negative differentiated cells (OCT4-). The bar graph represents the mean \pm SEM of 3 independent experiments. Student's 2-tailed $t$ test was used to determine significance: $* \mathrm{p}<0.05$. Magnification: $\times 60$.

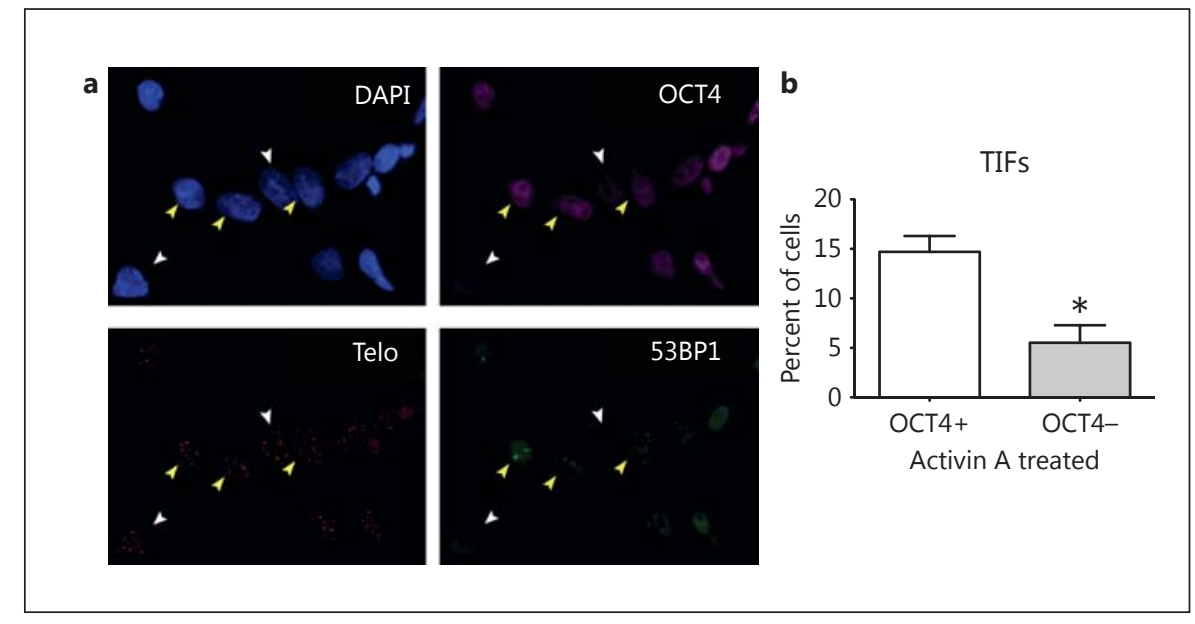

ogy (fig. 6d, top panel). However, Activin A treatment caused the UCSF4 cells to become flat and grow as a monolayer (fig. $6 \mathrm{~d}$, bottom panel), which is typical of differentiated cells. Immunofluorescent staining for the stem cell marker, OCT4, showed that $96 \%$ ( $\pm 2 \%$ SEM) of control UCSF4 cells were OCT4 positive, while only $35 \%$ $( \pm 4 \%$ SEM $)$ of the Activin A-treated UCSF4 cells were OCT4 positive (fig. 6e). A majority of the Activin A-treated UCSF4 cells had therefore differentiated, which would explain the increase in cells in G1 phase.

\section{Differentiated UCSF4 hESCs Have Fewer TIFs than Undifferentiated Cells}

Although Activin A-treated UCSF4 cells showed increased telomere dysfunction, the damage was not equally distributed among differentiated and undifferentiated cells. OCT4-positive undifferentiated cells were much more likely to be TIF positive than OCT4-negative differentiated cells (fig. 7). Because TIFs are more likely to occur in S phase of the cell cycle [Takai et al., 2003], this result suggests that OCT4-positive, undifferentiated cells are more likely to acquire telomere damage because they are still actively dividing. In contrast, because differentiated, OCT4-negative cells are growth-arrested, they no longer acquire telomere damage.

\section{Discussion}

Telomere dysfunction commonly occurs in cultured mammalian cells [Sabatier et al., 2005; Muraki et al., 2012]. We investigated whether UCSF4 cells experience telomere dysfunction when grown in culture and ob- served that UCSF4 cells grown in defined mTESR1 medium have an average of 7 dysfunctional telomeres per cell, which represents $\sim 4 \%$ of all telomeres. This percentage is very similar to that reported for mouse embryonic fibroblasts, in which $<5 \%$ of telomeres were found to be dysfunctional [Sfeir et al., 2009], but it is much less than that observed in BJ human primary fibroblasts, which exhibit more than $10 \%$ dysfunctional telomeres under standard conditions [Suram et al., 2012]. Experiments were performed using cells between passage 20 and 40. Although we observed no effect of the passage number on the extent of telomere dysfunction, we cannot rule out the possibility that the sensitivity to replication stress and telomere dysfunction may change at a higher or lower passage number.

Growth conditions can significantly affect replication stress and telomere dysfunction. It has been reported for various cell types that low levels of aphidicolin cause replication stress [Arlt et al., 2012] and can result in telomere dysfunction [Sfeir et al., 2009]. Our results show that aphidicolin also increases telomere dysfunction and TIF formation in UCSF4 cells in a dose-dependent manner. In contrast, addition of nucleosides reduced telomere dysfunction and TIF formation. Takai et al. [2003] observed that TIFs typically appear during $S$ phase, indicating that DNA replication promotes TIF formation. The ability of nucleosides to reduce TIFs in the UCSF4 cell line therefore demonstrates that UCSF4 grown in a defined medium can suffer from replication stress, which is at least partially due to insufficient nucleotide pools.

The growth factor Activin A has been described to have different effects on hESCs grown in culture. Laboratories have previously reported that when added at low levels (10 
$\mathrm{ng} / \mathrm{ml})$, Activin A maintains hESC proliferation and pluripotency by signaling through SMAD proteins [Beattie et al., 2005; Xiao et al., 2006]. However, other laboratories report that this level of Activin A can induce differentiation [Sulzbacher et al., 2009; Sa and McCloskey, 2012]. Our data demonstrate that in UCSF4 cells, Activin A induces telomere dysfunction as well as growth arrest and differentiation. One possible mechanism by which Activin A promotes DSBs and telomere dysfunction in UCSF4 cells is that, similar to oncogenes, Activin A can cause replication stress due to an increased rate of cell division. However, it is also possible that in UCSF4 cells Activin A somehow directly causes DSBs or inhibits DNA damage response (DDR) or DSB repair pathways. Regardless, our results demonstrate that the addition of some growth factors to hESC lines can promote telomere dysfunction.

There are several possible mechanisms by which Activin A might cause differentiation and growth arrest in UCSF4 cells. It is possible that Activin A signaling causes the down-regulation of genes responsible for pluripotency. There are conflicting studies in the literature, with some groups reporting that Activin A helps to maintain pluripotency in some hESC lines in culture [Beattie et al., 2005; Vallier et al., 2005; Xiao et al., 2006], while others report that Activin A promotes differentiation [Sulzbacher et al., 2009; Sa and McCloskey, 2012]. These differing results could be caused by differences in the hESC lines used, the concentration of Activin A, or the length of the Activin A treatment. Another possibility is that the effects of Activin A on differentiation are a result of the activation of DDR. Another group showed that p53, which is a critical player in DDR, causes differentiation and apoptosis in hESCs [Qin et al., 2007]. It is therefore possible that DDR resulting from Activin A-induced DSBs pushes the cells to differentiate. Differentiated cells, unlike undifferentiated cells, have a robust G1/S checkpoint [Sokolov and Neumann, 2012], which causes cells with DNA damage to arrest in G1 phase, preventing additional damage from forming during DNA synthesis. Differentiation and growth arrest also serve as mechanisms for tumor suppression by taking damaged cells out of the actively dividing population.

Although UCSF4 cells treated with Activin A have increased telomere dysfunction, OCT4-positive undifferentiated cells in the population were much more likely to be TIF-positive than OCT4-negative differentiated cells (fig. 7). Since TIFs are generated during DNA synthesis [Takai et al., 2003], a simple explanation is that the difference between the frequency of TIFs in the undifferenti-

Telomere Dysfuntion in Human

Embryonic Stem Cells ated and differentiated cells in the population is that the former divide more frequently than the latter. Regardless of the mechanism, these results demonstrate that the extent of the increase in telomere dysfunction caused by Activin A in undifferentiated UCSF4 cells is much higher than is indicated by our assay results, since our telomere dysfunction assay only measures cells in mitosis. The combined data therefore demonstrate that the extent of telomere dysfunction in the UCSF4 cell line during growth in culture can be either significantly increased or decreased by altering the factors present in their growth medium. It is therefore important to determine whether the effect of various factors on telomere dysfunction in UCSF4 cells is common to other hESC lines to optimize growth conditions to avoid chromosome instability during growth in culture.

We have demonstrated in this study that UCSF4 cells grown in a defined medium show telomere dysfunction. We have also demonstrated that changes in growth conditions, either through the addition of nucleosides or growth factors, can substantially influence the extent of this telomere dysfunction. Our results also suggest that this telomere dysfunction occurs through replication stress. As in the case of oncogene-induced replication stress, this replication stress in UCSF4 cells may be due to continuous cell division, because unlike hESCs in vivo, which only divide a few times during a human lifetime, UCSF4 cells, like other hESCs in culture, divide continuously. Regardless of the mechanism by which it occurs, minimizing telomere dysfunction in hESCs is important because it can result in chromosome instability, which can lead to cancer [Muraki et al., 2012]. Although it is well established that dysfunctional telomeres and telomere loss can lead to chromosome rearrangements and instability in other cell types, including mouse embryonic stem cells [Sabatier et al., 2005; Muraki et al., 2012], it has yet to be demonstrated whether telomere dysfunction leads to chromosome rearrangements in hESCs. Regardless, in view of the importance of telomeres for chromosome stability, hESCs grown in culture for therapeutic uses should be monitored for telomere dysfunction. The Q-FISH and TIF staining protocols, which are feasible using standard laboratory equipment, are therefore important tools for routinely monitoring telomere health and optimizing culture conditions of hESCs.

\section{Acknowledgements}

Funding for this project was provided by the California Institute of Regenerative Medicine grant RB3-05020. 


\section{References}

Arlt MF, Wilson TE, Glover TW: Replication stress and mechanisms of $\mathrm{CNV}$ formation. Curr Opin Genet Dev 22:204-210 (2012).

-Beattie GM, Lopez AD, Bucay N, Hinton A, Firpo MT, et al: Activin A maintains pluripotency of human embryonic stem cells in the absence of feeder layers. Stem Cells 23:489-495 (2005).

Bester AC, Roniger M, Oren YS, Im MM, Sarni D, et al: Nucleotide deficiency promotes genomic instability in early stages of cancer development. Cell 145:435-446 (2011).

- Bignell GR, Greenman CD, Davies H, Butler AP Edkins S, et al: Signatures of mutation and selection in the cancer genome. Nature 463: 893-898 (2010).

Burrell RA, McClelland SE, Endesfelder D, Groth $\mathrm{P}$, Weller MC, et al: Replication stress links structural and numerical cancer chromosomal instability. Nature 494:492-496 (2013).

-Capper R, Britt-Compton B, Tankimanova M, Rowson J, Letsolo B, et al: The nature of telomere fusion and a definition of the critical telomere length in human cells. Genes Dev 21: 2495-2508 (2007).

d'Adda di Fagagna F, Reaper PM, Clay-Farrace L, Fiegler H, Carr P, et al: A DNA damage checkpoint response in telomere-initiated senescence. Nature 426:194-198 (2003).

Felfly H, Klein OD: Sprouty genes regulate proliferation and survival of human embryonic stem cells. Sci Rep 3:2277 (2013).

-Fumagalli M, Rossiello F, Clerici M, Barozzi S, Cittaro D, et al: Telomeric DNA damage is irreparable and causes persistent DNA-damage-response activation. Nat Cell Biol 14:355365 (2012).

Krtolica A, Larocque N, Genbacev O, Ilic D, Coppe JP, et al: GROa regulates human embryonic stem cell self-renewal or adoption of a neuronal fate. Differentiation 81:222-232 (2011).
Lo AW, Sabatier L, Fouladi B, Pottier G, Ricoul M, Murnane JP: DNA amplification by breakage/fusion/bridge cycles initiated by spontaneous telomere loss in a human cancer cell line. Neoplasia 4:531-338 (2002).

Martínez P, Thanasoula M, Muñoz P, Liao C, Tejera A, et al: Increased telomere fragility and fusions resulting from TRF1 deficiency lead to degenerative pathologies and increased cancer in mice. Genes Dev 23:20602075 (2009).

Muraki K, Nyhan K, Han L, Murnane JP: Mechanisms of telomere loss and their consequences for chromosome instability. Front Oncol 2:135 (2012).

Murnane JP: Telomere loss as a mechanism for chromosome instability in human cancer. Cancer Res 70:4255-4259 (2010).

Nandakumar J, Cech TR: Finding the end: recruitment of telomerase to telomeres. Nat Rev Mol Cell Biol 14:69-82 (2013).

Poon, SS, Lansdorp PM: Quantitative fluorescence in situ hybridization (Q-FISH). Curr Protoc Cell Biol 18:18.4 (2001).

Qin H, Yu T, Qing T, Liu Y, Zhao Y: Regulation of apoptosis and differentiation by $\mathrm{p} 53 \mathrm{in} \mathrm{hu}$ man embryonic stem cells. J Biol Chem 282 5842-5852 (2007).

$\checkmark$ Sa S, McCloskey KE: Activin A and BMP4 signaling for efficient cardiac differentiation of $\mathrm{H} 7$ and H9 human embryonic stem cells. J Stem Cell Reg Med 8:198-202 (2012).

Sabatier L, Ricoul M, Pottier G, Murnane JP: The loss of a single telomere can result in instability of multiple chromosomes in a human tumor cell line. Mol Cancer Res 3:139-150 (2005).

Sfeir A, Kosiyatrakul ST, Hockemeyer D, MacRae SL, Karlseder J, et al: Mammalian telomeres resemble fragile sites and require TRF1 for efficient replication. Cell 138:90-103 (2009).
Sokolov MV, Neumann RD: Human embryonic stem cell responses to Ionizing radiation exposures: current state of knowledge and future challenges. Stem Cells Int 2012:579104 (2012).

Sulzbacher S1, Schroeder IS, Truong TT, Wobus AM: Activin A-induced differentiation of embryonic stem cells into endoderm and pancreatic progenitors - the influence of differentiation factors and culture conditions. Stem Cell Rev 5:159-173 (2009).

Suram A, Kaplunov J, Patel PL, Ruan H, Cerutti $A$, et al: Oncogene-induced telomere dysfunction enforces cellular senescence in human cancer precursor lesions. EMBO J 31: 2839-2851 (2012).

Takai H, Smogorzewska A, De Lange T: DNA damage foci at dysfunctional telomeres. Curr Biol 13:1549-1556 (2003)

Vallier L, Alexander M, Pedersen RA: Activin/ nodal and FGF pathways cooperate to maintain pluripotency of human embryonic stem cells. J Cell Sci 118:4495-4509 (2005).

-Wang L, Schulz TC, Sherrer ES, Dauphin DS, Shin S, et al: Self-renewal of human embryonic stem cells requires insulin-like growth factor-1 receptor and ERBB2 receptor signaling. Blood 110:4111-4119 (2007).

Xiao L, Yuan X, Sharkis SJ: Activin A maintains self-renewal and regulates fibroblast growth factor, WNT, and bone morphogenic protein pathways in human embryonic stem cells. Stem Cells 24:1476-1486 (2006).

Xu RH, Peck RM, Li DS, Feng X, Ludwig T, Thomson JA: Basic FGF and suppression of BMP signaling sustain undifferentiated proliferation of human ES cells. Nat Methods 2: 185-190 (2005).

Zeman MK, Cimprich K: Causes and consequences of replication stress. Nat Cell Biol 16: 2-9 (2014) 